\title{
Impact of Telemedicine on Patient Satisfaction and Perceptions of Care Quality in Radiation Oncology
}

Narek Shaverdian, MD ${ }^{1, *}$; Erin F. Gillespie, MD ${ }^{1, *}$; Elaine Cha, BS ${ }^{1}$; Soo Young Kim, MS²; Stephanie Benvengo, MS1; Fumiko Chino, MD1; Jung Julie Kang, MD, PhD'; Yuelin Li, PhD2,3; Thomas M. Atkinson, PhD²; Nancy Lee, MD; Charles M. Washington, $\mathrm{PhD}^{1}$; Oren Cahlon, $\mathrm{MD}^{1}$; and Daniel R. Gomez, $\mathrm{MD}^{1}$

\begin{abstract}
Background: The COVID-19 pandemic has transformed cancer care with the rapid expansion of telemedicine, but given the limited use of telemedicine in oncology, concerns have been raised about the quality of care being delivered. We assessed the patient experience with telemedicine in routine radiation oncology practice to determine satisfaction, quality of care, and opportunities for optimization. Patients and Methods: Patients seen within a multistate comprehensive cancer center for prepandemic office visits and intrapandemic telemedicine visits in December 2019 through June 2020 who completed patient experience questionnaires were evaluated. Patient satisfaction between office and telemedicine consultations were compared, patient visit-type preferences were assessed, and factors associated with an office visit preference were determined. Results: In total, 1,077 patients were assessed (office visit, $n=726$; telemedicine, $n=351$ ). The telemedicine-consult survey response rate was $40 \%$. No significant differences were seen in satisfaction scores between office and telemedicine consultations, including the appointment experience versus expectation, quality of physician's explanation, and level of physician concern and friendliness. Among telemedicine survey respondents, $45 \%$ and $34 \%$ preferred telemedicine and office visits, respectively, and $21 \%$ had no preference for their visit type. Most respondents found their confidence in their physician $(90 \%)$, understanding of the treatment plan (88\%), and confidence in their treatment $(87 \%)$ to be better or no different than with an office visit. Patients with better performance status and who were married/partnered were more likely to prefer in-person office visit consultations (odds ratio [OR], 1.04 [95\% Cl, 1.00-1.08]; $P=.047$, and 2.41 [95\% Cl, 1.14-5.47]; $P=.009$, respectively). Patients with telephone-only encounters were more likely to report better treatment plan understanding with an office visit $(\mathrm{OR}, 2.25 ; 95 \% \mathrm{Cl}$, $1.00-4.77 ; P=.04)$. Conclusions: This study is the first to assess telemedicine in routine radiation oncology practice, and found high patient satisfaction and confidence in their care. Optimization of telemedicine in oncology should be a priority, specifically access to audiovisual capabilities that can improve patient-oncologist communication.
\end{abstract}

J Natl Compr Canc Netw 2021;19(10):1174-1180 doi: $10.6004 /$ jnccn.2020.7687

${ }^{1}$ Department of Radiation Oncology, ${ }^{2}$ Department of Psychiatry and Behavior Sciences, and ${ }^{3}$ Department of Epidemiology and Biostatistics, Memorial Sloan Kettering Cancer Center, New York, New York.

*These authors contributed equally and are co-first authors.

\section{Background}

The coronavirus disease 2019 (COVID-19) pandemic has transformed cancer care in the United States with the rapid expansion of telemedicine into routine patient evaluation and management. ${ }^{1}$ Patients with cancer are vulnerable to COVID-19 because they are often older and have underlying comorbidities found to predict for poor COVID-19 outcomes, ${ }^{2-5}$ and therefore they may uniquely benefit from telemedicine. However, the perspectives and experiences of patients with telemedicine in everyday cancer care remain unknown and could have implications beyond the pandemic.

Prior to March 2020, telemedicine in the United States was primarily used to provide follow-up care to patients in remote locations and had additional regulations set by the Centers for Medicare \& Medicaid Services (CMS) that limited it use. ${ }^{6,7}$ Specifically, regulations limited telehealth to patients in a nonurban areas with established provider relationships and required visits to occur in a healthcare facility. ${ }^{1}$ Although previous studies have found high patient satisfaction with telemedicine, most studies are limited to these select patient populations. $^{8-10}$ Patients with cancer have strong informational needs and effective doctor-patient communication during oncologic consultation has been found to impact patient quality of life. ${ }^{11-13}$ Therefore, to ensure optimal patient outcomes it is necessary to assess patient satisfaction and quality of care with telemedicine given the rapid expansion of its use into routine cancer care.

We explored telemedicine in radiation oncology practice among patients in a comprehensive cancer center in the New York City metropolitan area, an early epicenter of the US COVID-19 pandemic, focusing on its use in consultations and on-treatment patient management. To optimize oncology practice during

See JNCCN.org for supplemental online content. 
the ongoing pandemic and to inform the postpandemic use of telemedicine, we compared patient satisfaction with prepandemic office visits versus telemedicine, assessed patient preferences for an office visit versus telemedicine, and identified factors that would predict for a patient preferring an in-person visit.

\section{Patients and Methods}

Patients and Interventions

This study assessed patients with cancer seen in radiation oncology clinics at Memorial Sloan Kettering Cancer Center (MSKCC), spanning 1 main campus and 6 regional centers across New York and New Jersey. The experience with telemedicine was evaluated across multiple phases of care, including the consultation appointment and weekly on-treatment management visits. To compare the telemedicine and in-person experience, 2 cohorts of patients, prepandemic and intrapandemic, were identified. The prepandemic period included patients seen in person from December 1, 2019, through February 28, 2020. The intrapandemic telemedicine period included patients seen via telemedicine from April 2, 2020, through June 10,2020 . As a result of COVID-19 mitigation, telemedicine was rapidly implemented in mid-March 2020 and became the default encounter for consultation visits. ${ }^{14,15}$ Telemedicine encounters used standard communication technologies, including telephone only or interactive audiovisual capabilities via smartphones or tablets/laptops/desktop computers. There were no specific policies dictating the need for an audiovisual versus telephone-only encounter, and appointment-type decisions were left to patients and providers. There were no specific restrictions on the physical location of patients or physicians during the telemedicine encounter.

\section{Instruments and Survey Administration}

Three instruments were used to assess prepandemic office visit consultations, telemedicine consultations, and telemedicine on-treatment management visits. A preexisting patient satisfaction survey with 12 questions assessed the in-person consultation experience and evaluated several domains, including appointment logistics, patient-physician communication, and overall impressions using a 5-point scale (top score $=5$ ). This preexisting patient satisfaction survey was developed by MSKCC and is used in active practice for quality improvement. The standard consultation survey was adapted in mid-March for telemedicine by removing questions specific to the in-office experience and adding questions querying patient preferences for telemedicine, office, or no preference to a series of prompts exploring patient-physician interactions, treatment plan understanding, appointment costs and logistics, and overall visit preference. Additional questions also explored concerns regarding the impact of COVID-19 on cancer care. The survey comprised a total of 20 questions. The telemedicine consult survey was developed based on literature review and input from content experts, and adapted questions from a previously published telemedicine survey that used measures developed by the Consumer Assessment of Healthcare Providers and Systems (CAHPS). ${ }^{16}$ To assess the telemedicine ontreatment management visit, the existing prepandemic on-treatment management visit patient satisfaction survey was similarly adapted. The surveys are provided in supplemental eAppendices 1-3 (available with this article at JNCCN.org).

All surveys were electronically administered by Quality Reviews as part of the standard quality improvement process. There was no patient selection, and all patients were sent a text message to the telephone number on record with a link to the electronic survey. No incentives were offered for survey completion. Two additional reminder messages were sent to initial nonrespondents. In total, 2,828 patients were surveyed (1,959 patients seen for prepandemic office visits and 870 seen for intrapandemic telemedicine visits). All patients who completed a survey were included in the analysis.

\section{Statistical Analysis}

All completed surveys were analyzed. There were no additional inclusion or exclusion criteria. A predefined statistical plan was used to minimize inflation of type I error. Patient characteristics and survey responses for office visit and telemedicine consultation patients were compared using chi-square or Fisher exact test. Logistic regression was used to identify patient and encounter variables, as shown in Table 1, associated with (1) an office visit overall preference (vs telemedicine or no preference), (2) improved treatment understanding with an office visit (vs telemedicine or no preference), and (3) concern that COVID-19 would impact the success of treatment. The telemedicine on-treatment management visit experience was assessed in patients who received radiotherapy (RT) after a telemedicine consultation. In patients with multiple on-treatment visits, longitudinal changes in survey responses were determined using the paired Wilcoxon and the Friedman test. All statistical associations were evaluated at a significance level of 0.05 (2-sided type I error rate). Analyses were conducted using $\mathrm{R}$ version 3.5.2 (R Foundation for Statistical Computing). This study was completed under an approved Institutional Review Board protocol. 


\begin{tabular}{|c|c|c|c|}
\hline Characteristic & $\begin{array}{l}\text { Office Visit } \\
n(\%)\end{array}$ & $\begin{array}{l}\text { Telemedicine } \\
\text { n (\%) }\end{array}$ & $\begin{array}{c}P \\
\text { Value }\end{array}$ \\
\hline Patients, N & 726 & 351 & \\
\hline Median age (range), y & $66(19-96)$ & 65 (19-91) & .05 \\
\hline Sex & & & .001 \\
\hline Female & $238(33)$ & $151(43)$ & \\
\hline Male & $488(67)$ & $200(57)$ & \\
\hline Race/Ethnicity ${ }^{a}$ & & & .70 \\
\hline White & $612(85)$ & $285(83)$ & \\
\hline Black & $41(6)$ & $26(8)$ & \\
\hline Asian & $31(4)$ & $14(4)$ & \\
\hline Hispanic & $42(6)$ & $24(7)$ & \\
\hline Other & $39(5)$ & $19(6)$ & \\
\hline Patient declined to answer & $17(2)$ & $8(2)$ & \\
\hline Karnofsky performance status & & & .06 \\
\hline$\leq 60$ & $27(4)$ & $5(2)$ & \\
\hline 70 & $26(4)$ & $14(4)$ & \\
\hline 80 & $174(25)$ & $65(21)$ & \\
\hline $90-100$ & $457(67)$ & $232(74)$ & \\
\hline Radiation consultation intent & & & .06 \\
\hline Palliative & $134(18)$ & $51(16)$ & \\
\hline Adjuvant & $202(28)$ & $120(34)$ & \\
\hline Definitive & $390(54)$ & $180(51)$ & \\
\hline Metastatic disease & & & .28 \\
\hline Yes & $170(23)$ & $72(21)$ & \\
\hline Symptomatic disease & & & .17 \\
\hline Yes & $86(12)$ & $52(15)$ & \\
\hline Cancer diagnosis category & & & .42 \\
\hline Prostate & $287(40)$ & $119(34)$ & \\
\hline Breast & $168(23)$ & $92(26)$ & \\
\hline Thoracic & $68(9)$ & $31(9)$ & \\
\hline Head and neck & $67(9)$ & $30(9)$ & \\
\hline Gastrointestinal & $48(7)$ & $29(8)$ & \\
\hline Other & $88(12)$ & $50(14)$ & \\
\hline
\end{tabular}

All missing values were excluded from calculating percentages and $P$ values. apatient selected and could identify in more than category.

\section{Results}

\section{Patient Characteristics}

We examined the experiences of 1,077 radiation oncology patients, including 351 patients seen for intrapandemic telemedicine visits and 726 seen for prepandemic office visits. The overall response rate was $38 \%$, and the response rate to the telemedicine consult survey was $40 \%$. Respondents and nonrespondents were similar in age $(P=.09)$ and sex $(P=.73)$. Table 1 shows respondent characteristics and group differences between patients who had telemedicine versus office visits. Patients with telemedicine visits had a median age of 65 years (range, $19-91$ years), $57 \%$ were male, and most $(74 \%)$ were married or partnered.

Telemedicine patients were largely evaluated for definitive $(51 \%)$ and adjuvant (34\%) RT, and the most common cancers included prostate $(34 \%)$ and breast (26\%). Median length of telemedicine consultation was 30 minutes (range, 15-80 minutes), and most patients ( $n=290$; $83 \%$ ) had an interactive audiovisual encounter, with the remainder $(\mathrm{n}=61 ; 17 \%)$ having a telephone-only visit.

\section{Patient Satisfaction With \\ Telemedicine Consultation}

Across all measured domains of patient satisfaction, no significant differences were found between telemedicine and office visit survey respondents with regard to radiation oncology consultation (Figure 1). Regardless of visit type, most survey respondents selected the top score when rating their actual appointment experience versus expectations (telemedicine: $82 \%$; office: $84 \%$ ), the quality of their physician's explanations (telemedicine: 91\%; office: $84 \%$ ), and the level of concern and friendliness shown by their physician (telemedicine: $92 \%$ and $95 \%$, respectively; office: $93 \%$ and $94 \%$, respectively). Similarly, $93 \%$ and $92 \%$ of telemedicine and office visit patients, respectively, selected the top score when rating their likelihood to recommend their physician to others. Additionally, no significant difference was found in mean patient satisfaction scores among survey respondents when assessed longitudinally across the prepandemic and intrapandemic periods (supplemental eFigure 1).

\section{Preferences Among Telemedicine \\ Consultation Patients}

Among patients who underwent a telemedicine consultation, when weighing all factors, $45 \%$ of survey respondents selected their overall appointment preference to be a telemedicine visit, $34 \%$ selected an office visit, and $21 \%$ found no difference between visit types (Figure 2). Most survey respondents selected either no difference or that telemedicine is better with regard to their confidence in their physician (90\%), understanding of their treatment plan (88\%), and confidence their cancer will be treated appropriately $(87 \%)$. Whereas most respondents (60\%), found no difference between visit types regarding the personal connection they feel with their physician, $30 \%$ selected an office visit as better for connection. Most survey respondents (91\%) selected no difference or telemedicine is preferred when sharing personal information. Although in terms of treatment-related costs $28 \%$ of survey respondents found no difference between visit types, most (66\%) preferred telemedicine (Figure 2). 


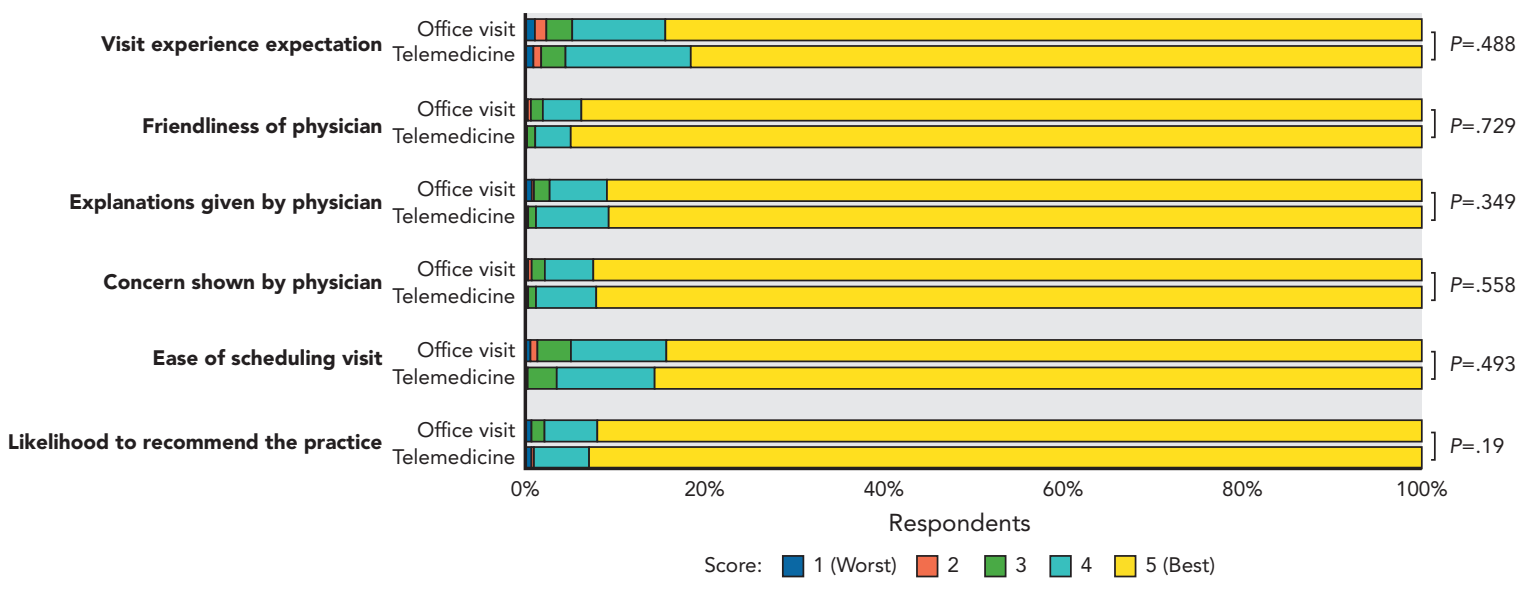

Figure 1. Patient satisfaction scores for prepandemic office visits and versus telemedicine consultations.

\section{Factors Associated With Preferring an Office Visit Consultation}

Across all patient and encounter-specific variables analyzed, only higher performance status (odds ratio [OR], $1.04 ; 95 \%$ CI, $1.00-1.08 ; P=.047)$ and being married/partnered (OR, 2.41; 95\% CI, 1.14-5.47; $P=.009$ ) were associated with a patient selecting an office visit as their overall visit preference [multivariate analysis presented here]. Age, sex, race/ethnicity, radiation consultation intent, cancer diagnosis, presence of symptomatic or metastatic disease, and length of visit were not associated with overall visit type preference (supplemental eTable 1). When assessing factors potentially influencing the outcome of understanding of the treatment plan, having a telephone-only visit was the sole factor associated with preference for an inperson office visit (OR, 2.25; 95\% CI, 1.00-4.77; $P=.040$ ).

\section{Longitudinal Assessment of Patient Experience} Through RT

Among patients completing a telemedicine consult survey $(n=351), 178$ received RT during the intrapandemic period, of whom $114(64 \%)$ also completed a telemedicine on-treatment management visit survey. Most respondents selected the top score across all satisfaction domains with regard to their on-treatment visit, including the quality of communication regarding treatment questions (92\%), preparedness for what to expect during treatment $(88 \%)$, and the quality of the visit with their physician (85\%). Forty-five patients completed at least 2 surveys allowing for a longitudinal assessment of the telemedicine experience. No significant differences in satisfaction scores or visit preference were found when comparing the first and last telemedicine on-treatment management visit (Figure 3).

\section{Patient Perspectives on the Impact of COVID-19 on Their Cancer Care}

Among patients who completed the telemedicine consultation survey $(n=351), 34 \%$ agreed that "cancer care will be negatively impacted" by the ongoing pandemic. However, only $8 \%$ expressed concern that their "cancer won't be successfully treated" as a result of the pandemic. Among respondents with an overall preference for a

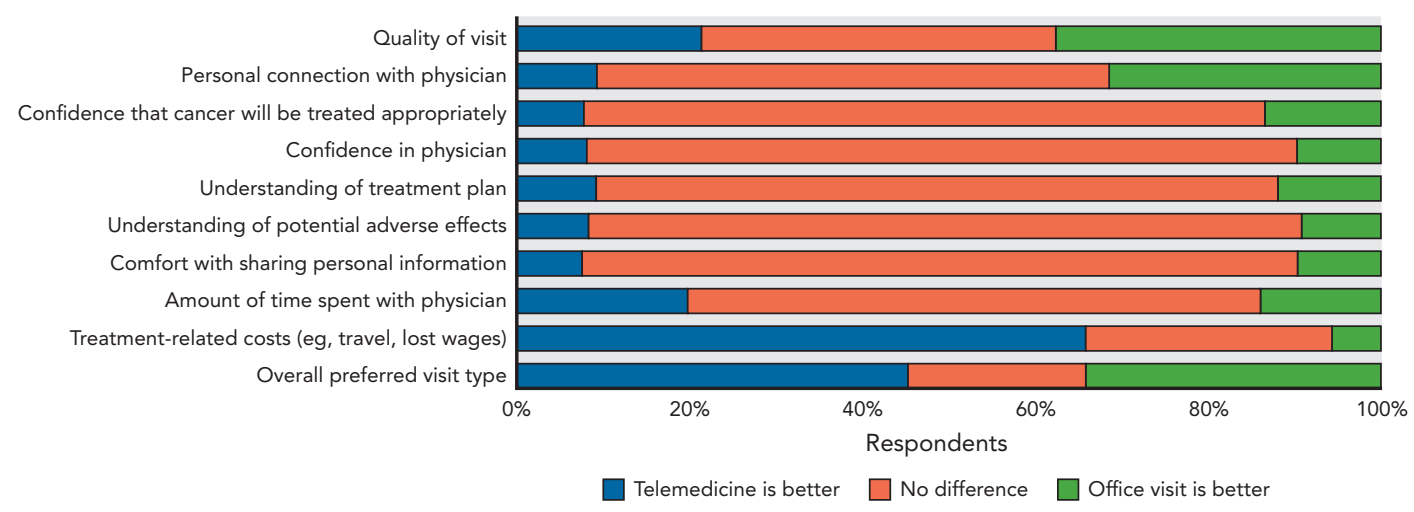

Figure 2. Preferences for an office visit versus telemedicine consultation. All surveyed patients had a telemedicine consultation. 


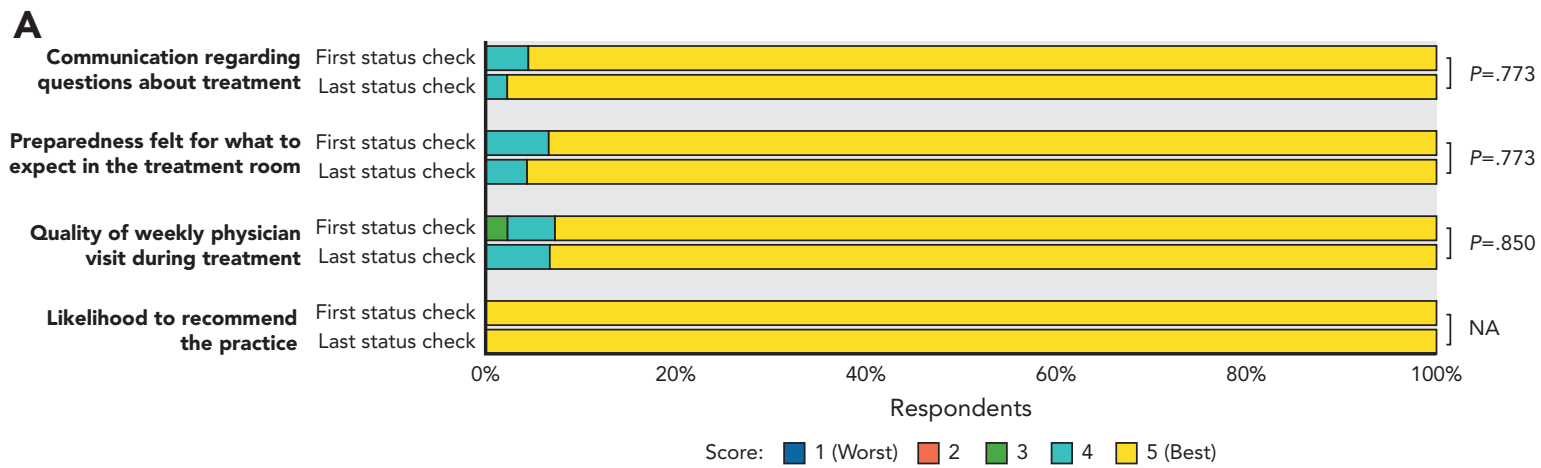

B

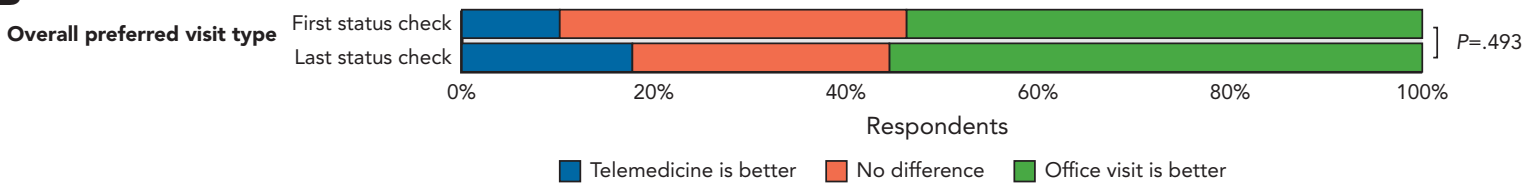

Figure 3. Patient assessment of the telemedicine on-treatment management visit. Data show comparison of (A) satisfaction scores and (B) visit preference type between the first and last on-treatment management visit.

telemedicine visit, $7 \%$ had concern that their treatment success would be negatively impacted by the pandemic versus $5 \%$ of respondents with an in-person office visit preference $(P=.64)$. No assessed factor was associated with patient concern that their cancer would not be successfully treated as a result of COVID-19. However, patients with prostate $(\mathrm{OR}, 0.21 ; 95 \% \mathrm{CI}, 0.06-0.66$; $P=.009$ ) or breast cancer (OR, 0.22 ; $95 \% \mathrm{CI}, 0.06-075$; $P=.02$ ) were significantly less likely to share concern that the pandemic would impact the success of their treatment compared with those with other cancer diagnoses.

\section{Discussion}

With COVID-19 instigating a rapid adoption of telemedicine in cancer care, we herein present, to our knowledge, the first robust assessment of the patients' perspective on this transformation of care delivery. First and foremost, we found no significant difference across all domains of patient satisfaction between prepandemic office visits and intrapandemic telemedicine visits among survey respondents. Most telemedicine survey respondents did not report inferior confidence in their physician, cancer treatment, or in their understanding of the treatment plan. Only 1 in 3 respondents selected office visit as their overall visit preference for their consultation, and satisfaction with telemedicine remained high as patients progressed through radiation treatment. Finally, our data suggest that interactive audiovisual encounters enhance patient-physician communication and improve patient understanding compared with telephone-only encounters. These data support the ongoing use of telemedicine in radiation oncology and offer insights to better optimize telemedicine for the future.

Prior to the COVID-19 pandemic, telemedicine had relatively low utilization and its use in oncology was mostly limited to patients in remote locations..$^{1,6,8,9,17}$ Patients with cancer generally desire a considerable amount of detail about their disease and treatment, and health professionals are the most frequent source of information. ${ }^{12,18}$ Additionally, the quality of a patient's interaction with their physician during their oncology consultation and effective physician-patient communication have a far-reaching impacts on patient wellbeing. ${ }^{11,19-21}$ Therefore, in the absence of widespread experience with telemedicine, concerns have been raised regarding its use in oncology. ${ }^{1}$ Multiple findings from the survey responses in our study serve to allay these concerns. Specifically, we found high patient satisfaction with telemedicine in the evaluation and management of patients with cancer in a major metropolitan area, a strong patient preference for telemedicine, and that nearly $90 \%$ of telemedicine respondents reported that their confidence in and understanding of their treatment were not inferior to those of an in-person office visit. Patient satisfaction in oncology is a multifactorial outcome that considers a range of factors, including interpersonal interactions, appointment length and efficiency, provider thoroughness, and perceptions of care coordination. ${ }^{22}$ Therefore, the high patient satisfaction with telemedicine despite the COVID-19 pandemic, wherein patients are facing increased vulnerability and myriad new stressors, ${ }^{23,24}$ further supports the use of telemedicine in oncology practice. 
Our data provide evidence that interactive audiovisual communication technologies enhance the quality of telemedicine encounters. Telephone-only patients were 2 times more likely than those who had an audiovisual encounter to report that their understanding of the treatment plan would be better with an in-person visit. Interestingly, no other examined variable, including patient age, performance status, appointment length, or cancer type, were associated with poor treatment plan understanding with telemedicine. Our finding regarding the importance of the audiovisual encounter is supported by multiple studies that have found that nonverbal interactions between clinicians and patients strengthen the therapeutic alliance and lead to higher patient satisfaction and quality of care. $^{25,26}$ This is potentially even more critical in oncology, where there is a high prevalence of psychologic distress, and nonverbal interactions have been found to be instrumental in eliciting and appreciating patient distress. ${ }^{25,27,28}$ Additionally, we found that one-third of all patients shared concerns about the negative impact of COVID-19 on cancer care. Therefore, telemedicine with audiovisual technologies must be prioritized during the ongoing pandemic to optimize communication and better preserve the patient-physician relationship amid this time of increased vulnerability.

Telemedicine can play an evolving role in cancer care as the pandemic continues and beyond the postpandemic recovery. Delays in the diagnosis and treatment of cancer as a result of COVID-19 have been modeled to result in tens to hundreds of thousands of excess cancer deaths. ${ }^{29-31}$ In a recent study, we found oncologists to report a high acceptance and ability to maintain quality of care with telemedicine. ${ }^{15}$ Therefore, given the favorable patient experience we report herein, telemedicine can uniquely facilitate the rapid evaluation of patients to prevent further delays in necessary cancer care, while reducing virus exposure. Perhaps unsurprisingly, we found that 2 out of 3 patients reported reduced treatment-related costs with telemedicine. Additionally, patients who were not married/partnered and those with a lower performance status were less likely to prefer an in-person visit, perhaps reflecting the increased burden of travel for those populations. Travel burden has been associated with decreased receipt of both chemotherapy $^{32}$ and radiation ${ }^{33}$ in curative cancer treatments, and can be an obstacle to receiving the best care with a complex cancer surgery. ${ }^{34}$ Given the efficiencies and cost savings of telemedicine, this method of care will be uniquely advantageous to patients both now and in the postpandemic era, assuming equitable access to the necessary technology is achieved. Recent data have indeed found disparities in access to digital technologies. Approximately one-quarter of Medicare beneficiaries lack either a smart phone or a computer with a high-speed internet connection, and without efforts to improve access to these technologies, the benefits of telemedicine will not translate to the most vulnerable patients. ${ }^{35}$

This study draws on the diverse experience of $>1,000$ patients with cancer treated in a comprehensive cancer center that spans 6 regional centers and provides vital data on telemedicine to inform oncology practice. However, this study has limitations. The pandemic itself and its impact on patients is a confounder. It has led to changes in regulations that have allowed for the widespread use of telemedicine, and it may have also impacted patient expectations of their healthcare providers and in the care they receive. Therefore, although we found no difference in the high patient satisfaction between the prepandemic and intrapandemic patients, these populations are not equivalent. Furthermore, although we assessed telemedicine through both consultation and on-treatment management and assessed for longitudinal changes in satisfaction, our data do not show the long-term perspectives of patients with their telemedicine-based care. Finally, although our response rate was adequate, limitations inherent to survey-based research pertaining to the survey population, the response-rate, and respondent bias also apply. Specifically, because this was an electronic survey conducted in an older adult patient population, selection bias as a result of digital literacy is of relevance, but we did not find a significant difference in age between respondents and nonrespondents. ${ }^{36}$ Although concern about COVID-19 did not appear to influence the preference for a telemedicine encounter, postpandemic studies assessing telemedicine perceptions will be necessary to confirm our findings. Additionally, studies focusing on diverse patient populations and qualitative data less prone to the ceiling effect are necessary.

\section{Conclusions}

This study provides a robust assessment of telemedicine in routine radiation oncology practice and finds high patient satisfaction and a strong preference for telemedicine among survey respondents. Telemedicine maintained patient confidence in the physician and treatment, but efforts to increase access to audiovisual technologies must be prioritized to improve patientphysician communication. These data should help inform efforts to maintain access to telemedicine for patients with cancer beyond the pandemic, while highlighting the importance of equitable access to audiovisual technologies to support the most vulnerable patients. Long-term assessment of cancer outcomes and further assessment of patient preferences are warranted. 


\section{Acknowledgments}

We are thankful to Sonni Mun, MD, at Quality Review for facilitating the data collection.

Submitted September 8, 2020; final revision received November 17, 2020; accepted for publication November 17, 2020. Published online January 4, 2021.

Author contributions: Study concept and design: Shaverdian, Gillespie, Gomez. Data acquisition: Shaverdian, Cha, Kim, Benvengo, Li, Atkinson. Data analysis and interpretation: All authors. Manuscript preparation: All authors. Critical revision: All authors. Administrative support: Washington, Cahlon, Gomez.
Disclosures: The authors have disclosed that they have not received any financial consideration from any person or organization to support the preparation, analysis, results, or discussion of this article.

Funding: Research reported in this publication was supported by the $\mathrm{NCl}$ of the NIH under award number P30 CA008748.

Disclaimer: The content is solely the responsibility of the authors and does not necessarily represent the official views of the NIH.

Correspondence: Narek Shaverdian, MD, Department of Radiation Oncology, Memorial Sloan Kettering Cancer Center, 1275 York Ave, New York, NY 10065. Email: shaverdn@mskcc.org

\section{References}

1. Royce TJ, Sanoff HK, Rewari A. Telemedicine for cancer care in the time of COVID-19. JAMA Oncol 2020;6:1698-1699.

2. Garassino MC, Whisenant JG, Huang LC, et al. COVID-19 in patients with thoracic malignancies (TERAVOLT): first results of an international, registry-based, cohort study. Lancet Oncol 2020;21:914-922.

3. Goyal P, Choi JJ, Pinheiro LC, et al. Clinical characteristics of COVID-19 in New York City. N Engl J Med 2020;382:2372-2374.

4. Luo J, Rizvi H, Preeshagul IR, et al. COVID-19 in patients with lung cancer. Ann Oncol 2020;31:1386-1396.

5. Lee LYW, Cazier JB, Angelis V, et al. COVID-19 mortality in patients with cancer on chemotherapy or other anticancer treatments: a prospective cohort study. Lancet 2020;395:1919-1926.

6. Harvey JB, Valenta S, Simpson K, et al. Utilization of outpatient telehealth services in parity and nonparity states 2010-2015. Telemedicine J E Health 2019;25:132-136.

7. Becker CD, Dandy K, Gaujean M, et al. Legal perspectives on telemedicine part 1: legal and regulatory issues. Perm J 2019;23:18-293.

8. Hamilton E, Van Veldhuizen E, Brown A, et al. Telehealth in radiation oncology at the Townsville Cancer Centre: service evaluation and patient satisfaction. Clin Transl Radiat Oncol 2018;15:20-25.

9. Taylor M, Khoo K, Saltman D, et al. The use of telemedicine to care for cancer patients at remote sites [abstract]. J Clin Oncol 2007;25(Suppl): Abstract 6538.

10. Allen A, Hayes J. Patient satisfaction with telemedicine in a rural clinic. Am J Public Health 1994;84:1693.

11. Ong LML, Visser MRM, Lammes FB, et al. Doctor-patient communication and cancer patients' quality of life and satisfaction. Patient Educ Couns 2000;41:145-156.

12. Jenkins V, Fallowfield L, Saul J. Information needs of patients with cancer: results from a large study in UK cancer centres. Br J Cancer 2001;84: 48-51.

13. Degner LF, Kristjanson LJ, Bowman D, et al. Information needs and decisional preferences in women with breast cancer. JAMA 1997;277: 1485-1492.

14. Kang JJ, Wong RJ, Sherman EJ, et al. The 3 Bs of cancer care amid the COVID-19 pandemic crisis: "be safe, be smart, be kind"-a multidisciplinary approach increasing the use of radiation and embracing telemedicine for head and neck cancer. Cancer 2020;126:4092-4104.

15. Zhang $\mathrm{H}$, Cha EE, Lynch $\mathrm{K}$, et al. Radiation oncologist perceptions of telemedicine from consultation to treatment planning: a mixed-methods study. Int J Radiat Oncol Biol Phys 2020;108:421-429.

16. Donelan K, Barreto EA, Sossong S, et al. Patient and clinician experiences with telehealth for patient follow-up care. Am J Manag Care 2019;25:40-44.

17. Humer MF, Campling BG. The role of telemedicine in providing thoracic oncology care to remote areas of British Columbia. Curr Oncol Rep 2017; 19:52.

18. Rutten LJ, Arora NK, Bakos AD, et al. Information needs and sources of information among cancer patients: a systematic review of research (1980-2003). Patient Educ Couns 2005;57:250-261.
19. Bensing J. Doctor-patient communication and the quality of care. Soc Sci Med 1991;32:1301-1310.

20. Stewart MA. Effective physician-patient communication and health outcomes: a review. CMAJ 1995;152:1423-1433.

21. Woolley FR, Kane RL, Hughes CC, et al. The effects of doctor-patient communication on satisfaction and outcome of care. Soc Sci Med 1978; 12:123-128.

22. Lis CG, Rodeghier M, Gupta D. Distribution and determinants of patient satisfaction in oncology: a review of the literature. Patient Prefer Adherence 2009;3:287-304.

23. Cook KA, Kahn JM. Distancing without isolating-connection in the era of COVID-19. JAMA Oncol 2020;6:1695

24. Gharzai LA, Resnicow K, An LC, et al. Perspectives on oncology-specific language during the coronavirus disease 2019 pandemic: a qualitative study. JAMA Oncol 2020;6:1424-1428.

25. Mast MS. On the importance of nonverbal communication in the physician-patient interaction. Patient Educ Couns 2007;67:315-318.

26. DiMatteo MR, Taranta A, Friedman HS, et al. Predicting patient satisfaction from physicians' nonverbal communication skills. Med Care 1980; 18:376-387.

27. Holland JC, Andersen B, Breitbart WS, et al. Distress management. J Natl Compr Canc Netw 2013;11:190-209.

28. Bensing JM, Kerssens JJ, van der Pasch M. Patient-directed gaze as a too for discovering and handling psychosocial problems in general practice. J Nonverbal Behav 1995;19:223-242.

29. Sud A, Jones ME, Broggio J, et al. Collateral damage: the impact on outcomes from cancer surgery of the COVID-19 pandemic. Ann Oncol 2020;31:1065-1074.

30. Maringe C, Spicer J, Morris M, et al. The impact of the COVID-19 pandemic on cancer deaths due to delays in diagnosis in England, UK: a national, population-based, modelling study. Lancet Oncol 2020;21: 1023-1034.

31. Sharpless NE. COVID-19 and cancer. Science 2020;368:1290.

32. Lin CC, Bruinooge SS, Kirkwood MK, et al. Association between geographic access to cancer care, insurance, and receipt of chemotherapy: geographic distribution of oncologists and travel distance. J Clin Oncol 2015;33:3177-3185.

33. Lin CC, Bruinooge SS, Kirkwood MK, et al. Association between geographic access to cancer care and receipt of radiation therapy for rectal cancer. Int J Radiat Oncol Biol Phys 2016;94:719-728.

34. Resio BJ, Chiu AS, Hoag JR, et al. Motivators, barriers, and facilitators to traveling to the safest hospitals in the United States for complex cancer surgery. JAMA Netw Open 2018;1:e184595.

35. Roberts ET, Mehrotra A. Assessment of disparities in digital access among medicare beneficiaries and implications for telemedicine. JAMA Intern Med 2020;180:1386-1389.

36. Lam K, Lu AD, Shi $Y$, et al. Assessing telemedicine unreadiness among older adults in the United States during the COVID-19 pandemic. JAMA Intern Med 2020;180:1389-1391. 
Supplemental online content for:

\section{Impact of Telemedicine on Patient Satisfaction and Perceptions of Care Quality in Radiation Oncology}

Narek Shaverdian, MD; Erin F. Gillespie, MD; Elaine Cha, BS; Soo Young Kim, MS; Stephanie Benvengo, MS; Fumiko Chino, MD; Jung Julie Kang, MD, PhD; Yuelin Li, PhD; Thomas M. Atkinson, PhD; Nancy Lee, MD; Charles M. Washington, PhD; Oren Cahlon, MD; and Daniel R. Gomez, MD

J Natl Compr Canc Netw 2021;19(10):1174-1180

eFigure 1: Longitudinal Assessment of Consultation Visit Satisfaction Scores

eTable 1: Bivariate Analysis for Selecting In-Person Office Visit

eAppendix 1: Office Visit Consultation Survey

eAppendix 2: Telemedicine Consultation Survey

eAppendix 3: Telemedicine On-Treatment Management Survey 


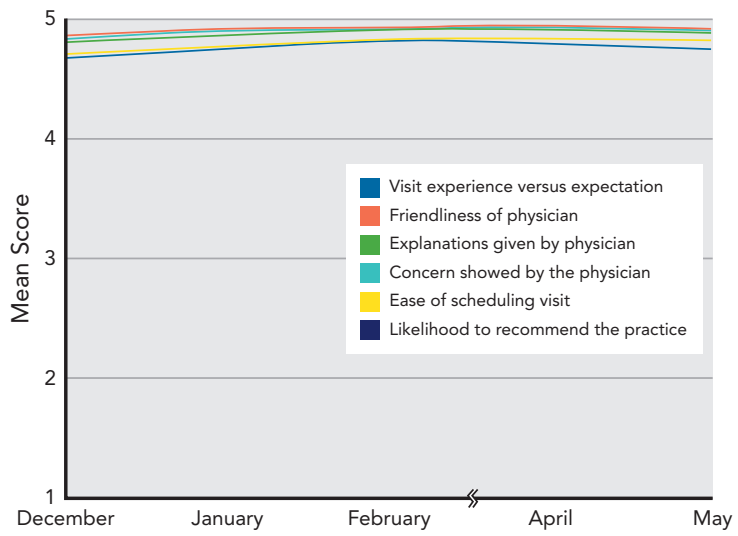

eFigure 1. Longitudinal assessment of consultation visit satisfaction scores measured across the prepandemic and intrapandemic periods. 


\section{eTable 1. Bivariate Analysis for Selecting In-Person Office Visit}

\begin{tabular}{|c|c|c|c|c|}
\hline Variable & \multicolumn{2}{|c|}{ Overall Preference $^{a}$} & \multicolumn{2}{|c|}{ Treatment Plan Understanding ${ }^{a}$} \\
\hline Age & $1.02(0.99-1.04)$ & .16 & $1.01(0.98-1.04)$ & .43 \\
\hline Karnofsky performance score & $1.04(1.00-1.08)$ & .04 & $1.00(0.96-1.04)$ & .92 \\
\hline \multicolumn{5}{|l|}{ Sex } \\
\hline Female & Ref & & Ref & \\
\hline \multicolumn{5}{|l|}{ Marital status } \\
\hline Married/Partnered & $2.26(1.11-4.89)$ & .03 & $1.00(0.48-2.25)$ & .99 \\
\hline Race/Ethnicity & & .96 & & .28 \\
\hline Black & $1.11(0.15-10.14)$ & & $0.35(0.05-2.27)$ & \\
\hline White & $1.07(0.20-7.88)$ & & $0.29(0.08-1.39)$ & \\
\hline Cancer diagnosis & & .98 & & .53 \\
\hline Prostate & $1.10(0.46-2.72)$ & & $1.23(0.40-4.64)$ & \\
\hline Breast & $0.95(0.37-2.51)$ & & $1.29(0.39-4.99)$ & \\
\hline Head and neck & $0.95(0.26-3.27)$ & & $2.44(0.59-10.76)$ & \\
\hline Thoracic & $0.79(0.20-2.83)$ & & $2.69(0.70-11.41)$ & \\
\hline Gastrointestinal & $0.81(0.23-2.74)$ & & $1.61(0.29-7.99)$ & \\
\hline Other & Ref & & Ref & \\
\hline \multicolumn{5}{|l|}{ Metastatic disease } \\
\hline Yes & $1.40(0.73-2.67)$ & .31 & $1.48(0.65-3.15)$ & .32 \\
\hline \multicolumn{5}{|l|}{ Telemedicine encounter type } \\
\hline Audiovisual & Ref & & Ref & \\
\hline Telephone only & $2.03(0.72-5.73)$ & .17 & $2.25(1.00-4.77)$ & .04 \\
\hline Appointment length & $1.01(0.99-1.02)$ & .52 & $1.01(0.99-1.03)$ & .28 \\
\hline
\end{tabular}

Bold indicates statistically significant values.

Abbreviation: OR, odds ratio.

${ }^{a}$ Association between factors and an in-person office visit preference (ref: telemedicine visit/no difference). 


\section{eAppendix 1. Office Visit Consultation Survey}

\section{On a scale of 1 (worst) to 5 (best):}

1. How would you rate the courtesy and communication shown by the clerks and receptionists upon your arrival to the department?

2. How would you rate how close your experience was compared with the expectation communicated by our office prior to your visit?

3. How would you rate the friendliness of the physician?

4. How would you rate the friendliness of the nurses and clinical staff?

5 . How would you rate the explanation the physician gave you about your condition?

6. How would you rate the concern the physician showed for your questions or concerns?

7. How would you rate the concern the nurses and clinical staff showed for your questions or worries?

8. How would you rate the staff's efforts to include you in decisions about appointment scheduling, during your consultation?

9. How would you rate the ease with which you were able to schedule today's visit?

10. How would you rate the waiting time during today's visit?

11. How likely are you to recommend our practice to others?

12. Please provide any additional comments.

\section{eAppendix 2. Telemedicine Consultation Survey}

\section{On a scale of 1 (worst) to 5 (best):}

1. How would you rate how close your experience was to the expectation communicated by our office prior to your visit?

2. How would you rate the friendliness of the physician?

3. How would you rate the explanation the physician gave you about your condition?

4. How would you rate the concern the physician showed for your questions or concerns?

5 . How would you rate the ease with which you were able to schedule today's visit?

6. I could see my doctor clearly. [Yes; No; Does not apply to me]

7. Compared with office visits, how would you rate the overall quality of the visit?

8. Compared with office visits, how would you rate the personal connection you felt with the doctor?

9. Compared with office visits, how would you rate your confidence that your cancer will be treated appropriately?

10. Compared with office visits, how would you rate confidence in your doctor?

11. Compared with office visits, how would you rate how well you understand your treatment plan?

12. Compared with office visits, how would you rate how well you understand potential side effects?

13. Compared with office visits, how would you rate how comfortable you feel about sharing personal information?

14. Compared with office visits, how would you rate the amount of time you spent with your clinician?

15. How would you compare your treatment-related costs (including travel and time off work)? [Telemedicine is better; No difference; Office visit is better; Does not apply to me]

16. When taking account all of the factors associated with your visit (time, cost, expense, convenience, quality of care, interaction with clinical team), what type of appointment would you have preferred? [Telemedicine is better; No difference; Office visit is better; Does not apply to me] 
17. I am concerned my cancer care will be negatively impacted by the COVID-19 pandemic. [Strongly agree; Agree; Neutral; Disagree; Strongly disagree]

18. I am concerned my cancer won't be successfully treated because of the COVID-19 pandemic. [Strongly agree; Agree; Neutral; Disagree; Strongly disagree]

19. How likely are you to recommend our practice to others?

20. Please provide any additional comments.

\section{eAppendix 3. Telemedicine On-Treatment Management Survey}

\section{On a scale of 1 (worst) to 5 (best):}

1. How would you rate the courtesy and communication shown by the clerks and receptionists upon your arrival to the department for treatment?

2. How would you rate our communication of wait time for your treatment by the staff?

3. How would you rate our communication regarding any questions you had about your treatment by the staff?

4. How well prepared do you feel for what to expect in the treatment room?

5 . How would you rate the friendliness of the radiation therapist team?

6. How would you rate the wait time of your daily radiation treatments?

7. How would you rate our communication about the need for weekly visits with the physician during treatment?

8. How would you rate the quality of the weekly visit with your physician during treatment?

9. How would you rate our communication of changes in your treatment schedule?

10. When taking account all of the factors associated with your visit (time, cost, expense, convenience, quality of care, interaction with clinical team), what type of appointment would you have preferred? [Telemedicine is better; No difference; Office visit is better; Does not apply to me]

11. How likely are you to recommend our practice to others?

12. Please provide any additional comments. 